\title{
AN APPLICATION OF TOPOLOGY OPTIMISATION TO DEFECT IDENTIFICATION IN TWO-DIMENSIONAL ELASTODYNAMICS WITH THE BEM AND H-MATRIX METHOD
}

\author{
KEI MATSUSHIMA, HIROSHI ISAKARI, TORU TAKAHASHI \& TOSHIRO MATSUMOTO \\ Nagoya University, Japan.
}

\begin{abstract}
This paper presents a numerical method for topology optimisation for two-dimensional elastodynamics based on the level set method and the boundary element method (BEM) accelerated by the $\mathcal{H}$-matrix method and its application to identifications of defects in an infinite elastic medium. Gradient-based topology optimisation methods require design sensitivity, which is obtained by solving some boundary value problems. The BEM is employed for this sensitivity analysis because the BEM can deal with infinite domains rigorously without any approximation. However, the computational cost in the BEM is expensive, and this is a serious drawback since we need to repeat sensitivity analysis even for a single optimisation process. In this study, the $\mathcal{H}$-matrix method is used as an acceleration method of the BEM for the reduction of the computational cost of the sensitivity analysis. Also proposed is a method to improve the efficiency of the $\mathcal{H}$-matrix method by exploiting a property of the kernel function of the elastodynamic fundamental solution. Some numerical examples are demonstrated, and the effectiveness of the proposed method is confirmed.

Keywords: boundary element method, defect identification, elastic wave, level set method, topological derivative, topology optimisation, $\mathcal{H}$-matrix method
\end{abstract}

\section{INTRODUCTION}

Recently, non-destructive testing has attracted attention in engineering, and a number of works have been made to develop an effective method to image defects in an elastic medium from some measured data [1]. To obtain such data, elastic waves are often used. For example, an elastic wave propagates in an elastic medium and is scattered by a defect. The scattered wave can then be measured as the displacement on some part of the boundary of the elastic medium. This is a so-called inverse scattering problem and can be treated as a topology optimisation problem with an elastic wave propagation phenomenon detecting the unknown defect shape and location. The objective functional of the corresponding optimisation problem is often defined to minimise a discrepancy between the measured data and solutions of the wave problem in the elastic domain with estimated defects. Topological derivative, which is defined as a rate of change of the objective functional (in this case, the discrepancy) when an infinitesimal defect is allocated in the medium, is useful for this approach. Some topological derivatives in three-dimensional elastodynamics are derived in $[2,3]$. The adjoint variable method is often used for the computation of topological derivatives, where we have to solve two kinds of boundary value problems: the direct and adjoint problems. For the analyses of these problems, the boundary element method (BEM) is preferred to the finite element method for a certain class of the problems because the BEM can deal with the infinite domain rigorously.

The computational cost of the BEM is highly expensive, therefore, the BEM is usually implemented with an acceleration method such as the fast multipole method (FMM) [4, 5] and $\mathcal{H}$-matrix method [6]. In the present optimisation scheme, a direct solver based on 
LU-decomposition is more effective than iterative solvers such as GMRES because both the coefficient matrices corresponding to the direct and adjoint problems are the same, thus LU-decomposition is more efficient than iterative solvers. In fact, we employed the $\mathcal{H}$-matrix method for a topology optimisation problem for two-dimensional electromagnetic field, and we demonstrated its effectiveness in [7].

In this study, we employ the $\mathcal{H}$-matrix method as an acceleration method of the BEM and apply it to the defect identification based on the topology optimisation with the level set method.

\section{TOPOLOGY OPTIMISATION WITH THE LEVEL SET METHOD}

2.1 Inverse scattering problem and corresponding topology optimisation problem

In the present study, we assume that all defects are assumed to be cavities having traction-free surfaces. Let $\Omega \subset \mathrm{R}^{2}$ be an infinite domain of a linear isotropic elastic medium under plane strain condition. As shown in Fig. 1, let us assume that an incident elastic wave $u^{\text {in }}$ propagates in $\Omega$ and is scattered by the defects $\mathrm{R}^{2} \backslash \bar{\Omega}$. Assuming time harmonic oscillations with the time dependence of $\mathrm{e}^{-\mathrm{i} \omega t}$, where $\omega$ is the angular frequency, the displacement $u$ and stress $\sigma$ in $\Omega$ are governed by the following boundary value problem:

$$
\begin{gathered}
\sigma_{j i, j}(x)+\rho \omega^{2} u_{i}(x)=0 \quad x \in \Omega, \\
t_{i}(x)=\sigma_{j i}(x) n_{j}(x)=0 \quad x \in \Gamma, \\
\text { Radiation condition for } u^{\mathrm{sc}} \text { as }|x| \rightarrow \infty,
\end{gathered}
$$

where $\rho$ is the mass density, $\Gamma=\partial \Omega$ is the boundary of $\Omega, u^{\mathrm{sc}}=u-u^{\mathrm{in}}$ is the scattered field, $t$ is the traction, and $n$ is the unit outward normal vector to $\Omega$.

Suppose that we measure the displacements on some observation points $x_{m}^{\text {obs }}(m=1, \ldots, M)$ and identify the distribution of the defects from the measured displacements $\hat{u}\left(x_{m}^{\mathrm{obs}}\right)$. This inverse scattering problem can be treated as a topology optimisation problem minimising the objective functional:

$$
J=\frac{1}{2} \sum_{m=1}^{M}\left|u\left(x_{m}^{\mathrm{obs}}\right)-\hat{u}\left(x_{m}^{\mathrm{obs}}\right)\right|^{2} .
$$

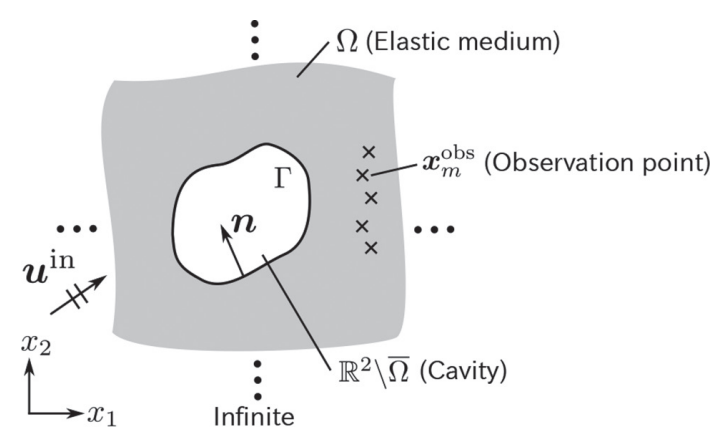

Figure 1: Problem statement. 


\subsection{Level set method}

For simplicity, we assume $\mathrm{R}^{2} \backslash \bar{\Omega}=D \backslash \bar{\Omega}$, i.e., there exists no defect outside of a certain bounded fixed design domain $D$. To express the distribution of the domains $\Omega$ and $D \backslash \bar{\Omega}$, we employ the level set method in which the defects $D \backslash \bar{\Omega}$, elastic medium $\Omega$, and its boundary $\Gamma$ are expressed by a level set function $\phi$, as follows:

$$
\begin{gathered}
\Omega=\{x \mid 0<\phi(x) \leq 1\}, \\
\Gamma=\{x \mid \phi(x)=0\}, \\
D \backslash \bar{\Omega}=\{x \mid-1 \leq \phi(x)<0\},
\end{gathered}
$$

The level set method converts the topology optimisation problem into a problem in which we find $\phi$ that minimises the objective functional $J$. We assume that $\phi$ is the solution of the following initial-boundary value problem $[8,9]$ :

$$
\begin{gathered}
\frac{\partial \phi(x, t)}{\partial t}=-K T(x, t)+\tau \nabla^{2} \phi(x, t) \quad x \in D, \\
\phi(x, 0)=\phi_{0} \quad x \in D, \\
\phi(x, t)=c \quad x \in \partial D
\end{gathered}
$$

where $K$ is a positive constant, $t$ is a fictitious time, $\tau$ is a positive constant that works as a regularisation parameter for the distribution of $\phi$, and $\phi_{0}$ is the initial distribution of $\phi$ corresponding to the initial geometry at $t=0$. The Dirichlet condition (10) with a constant $c \in(0,1]$ restricts that the defects can only exist inside $D$. $T$ denotes the topological derivative, defined by

$$
\delta J(x)=T(x) \nu(\varepsilon)+o(\nu(\varepsilon)),
$$

where $\delta J(x)$ is the variation of $J$ when an cylindrical defect of radius $\varepsilon$ centred at $x$ appears and $v$ is a scalar function which vanishes as $\varepsilon \rightarrow 0$. For the objective function $J$ defined by (4), $T$ is obtained as

$$
T(x)=\operatorname{Re}\left[\frac{\lambda+2 \mu}{4 \mu(\lambda+\mu)}\left\{4 \sigma_{i j}(x) \tilde{\sigma}_{i j}(x)-\sigma_{i i}(x) \tilde{\sigma}_{j j}(x)\right\}-\rho \omega^{2} u_{i}(x) \tilde{u}_{i}(x)\right]
$$

where $\tilde{u}$ and $\tilde{\sigma}$ are the displacement and stress, respectively, of the adjoint field problem governed by

$$
\begin{gathered}
\tilde{\sigma}_{j i, j}(x)+\rho \omega^{2} \tilde{u}_{i}(x)+\sum_{m=1}^{M} \overline{\left(u_{i}\left(x_{m}^{\mathrm{obs}}\right)-\hat{u}_{i}\left(x_{m}^{\mathrm{obs}}\right)\right)} \delta\left(x-x_{m}^{\mathrm{obs}}\right)=0 \quad x \in \Omega, \\
\tilde{t}_{i}(x)=\tilde{\sigma}_{j i}(x) n_{j}(x)=0 \quad x \in \Gamma,
\end{gathered}
$$

Radiation condition for $\tilde{u}_{i}(x)$ as $|x| \rightarrow \infty$. 
2.3 Algorithm of the level set-based topology optimisation

As described in the previous section, we solve the initial-boundary value problem (8)-(10) to obtain the optimal configuration. In this study, we perform the topology optimisation by the following procedure:

1. Determine an initial configuration and initialise a level set function $\phi$. The values of both $\phi$ and the topological derivative $T$ are computed and stored at all the lattice points arranged in the fixed design domain $D$.

2. Interpolate $\phi$ linearly between each adjacent lattice point and calculate the point of intersection between the segment of two neighboring lattice points and the zero-valued isoline of $\phi$. Then, connect these intersection points of $\phi$ and interpolate them with the Hermite spline curve. Finally, divide the generated isoline curve into linear boundary elements of approximately equal lengths [10].

3. Solve the direct problem (1)-(3) by the BEM.

4. Compute the value of the objective function $J$ and the gradient of $J$. If the absolute value of the gradient is less than a preset parameter, terminate the procedure; otherwise, go to 5 .

5. Solve the adjoint problem by the BEM and compute the topological derivative $T$ from the numerical solutions of the direct and adjoint problems.

6. Update $\phi$ according to (8)-(10) and go to 2 .

The entire step consisting of the steps 2 to 6 is called as an optimisation step in this paper.

\section{SENSITIVITY ANALYSIS WITH THE BEM AND $\mathcal{H}$-MATRIX METHOD}

\subsection{Formulation of the BEM in two-dimensional elastodynamics}

In order to compute the topological derivative $T$, we need to obtain both the displacement and stress in the direct and adjoint fields governed by (1)-(3) and (13)-(15), respectively.

We obtain the boundary integral equations equivalent to the direct problem (1)-(3) by the Burton-Miller formulation [11] as follows:

$$
\left[\begin{array}{cc}
\frac{1}{2} I+D_{11}+\alpha N_{11} & D_{12}+\alpha N_{12} \\
D_{21}+\alpha N_{21} & \frac{1}{2} I+D_{22}+a N_{22}
\end{array}\right]\left(\begin{array}{l}
u_{1} \\
u_{2}
\end{array}\right)=\left(\begin{array}{c}
u_{1}^{i n}+\alpha t_{1}^{i n} \\
u_{2}^{i n}+\alpha t_{2}^{i n}
\end{array}\right),
$$

where $\alpha \in \mathrm{C}$ is a coupling parameter, $I$ is the identity operator, $D_{i j}$ and $N_{i j}$ are the integral operators defined for an arbitrary scalar function $w$ as

$$
\begin{gathered}
\left(D_{i j} w\right)(x)=- \text { v.p. } \int_{\Gamma} C_{k l j m} G_{k i, l}(x, y) n_{m}(y) w(y) d \Gamma_{y}, \\
\left(N_{i j} w\right)(x)=- \text { p.f. } \int_{\Gamma} C_{i m p q} C_{k l j n} G_{k p, l q}(x, y) n_{m}(x) n_{n}(y) w(y) d \Gamma_{y},
\end{gathered}
$$

where v.p. and p.f. indicate Cauchy's principal value and the finite part of divergent integral, respectively. $C$ is the elasticity tensor and is written using Kronecker's delta $\delta_{i j}$ and Lamé's constants $\lambda$ and $\mu$ as 


$$
C_{i j k l}=\lambda \delta_{i j} \delta_{k l}+\mu\left(\delta_{i k} \delta_{j l}+\delta_{i l} \delta_{j k}\right),
$$

and $G_{i j}$ is the fundamental solution of two-dimensional elastodynamic problems given by

$$
G_{i j}(x, y)=\frac{i}{4 \mu}\left\{H_{0}^{(1)}\left(k_{\mathrm{T}}|x-y|\right) \delta_{i j}+\frac{1}{k_{\mathrm{T}}^{2}} \frac{\partial^{2}}{\partial y_{i} \partial y_{j}}\left(H_{0}^{(1)}\left(k_{\mathrm{T}}|x-y|\right)-H_{0}^{(1)}\left(k_{\mathrm{L}}|x-y|\right)\right)\right\},
$$

where $H_{0}^{(1)}$ is the Hankel function of first kind with order 0 and $k_{\mathrm{L}}$ and $k_{\mathrm{T}}$ are the wavenumbers of the longitudinal and transverse wave, respectively.

\section{$3.2 \mathcal{H}$-matrix method}

As described in Section 2.3, iterative sensitivity analyses are required for our optimisation method, causing significantly large computation time. In this study, we employ the $\mathcal{H}$-matrix method to the BEM and accelerate the sensitivity analyses.

The algorithm of the $\mathcal{H}$-matrix method is composed of two processes: hierarchical blocking of a coefficient matrix into submatrices and low rank approximation of the submatrices.

\subsubsection{Hierarchical blocking of a coefficient matrix}

Let us consider a system of linear algebraic equations which is obtained by discretising the BIEs (16) by the collocation method with constant elements. In the $\mathcal{H}$-matrix method, a set of boundary elements is called a cluster. Let $I$ be the set of all the boundary elements and obtain a binary tree $T$ whose root cluster is $I$ by the following recursive procedure:

1. Set $C=I$.

2. If the number of elements which are included in $C$ is larger than a preset parameter $n_{\text {min }}$, then go to 3; otherwise set $C$ to be a leaf cluster, which has no son cluster.

3. Make a rectangle which encloses all elements in $C$.

4. Divide the longer side of the rectangle into two parts of equal size. Correspondingly, $C$ is also divided into two subsets $C_{1}$ and $C_{2}$ so that each subset consists of elements whose collocation point is included in one of the two rectangles.

5. Renumber the elements so that the elements in the same cluster have the element numbers close to each other.

6. Set $C_{1}$ and $C_{2}$ to be son clusters of $C$.

7. For $i=1 ; 2$, set $C=C_{i}$ and go to 2 .

Next, we consider the blocking procedure of the coefficient matrix in (16). For illustrative purpose, we pick one of the four submatrices in (16). We divide the matrix into some submatrices according to the cluster and classify them into admissible and inadmissible blocks, which will be low lank-approximated or not, respectively, by the following procedure:

1. Set $C_{r}=I$ and $C_{c}=I$.

2. If $C_{r}$ and $C_{c}$ satisfy the admissible condition defined as

$$
\min \left\{\operatorname{diam} C_{r}, \operatorname{diam} C_{c}\right\} \leq \eta \operatorname{dist}\left(C_{r}, C_{c}\right),
$$

where $\eta \in \mathrm{R}$ is a preset parameter and 'diam' and 'dist' denote a diameter and distance of a cluster, respectively, then set the submatrices whose row indices and column indices correspond to $C_{r}$ and $C_{c}$ to be admissible blocks, respectively; otherwise, go to 3 
3. If both $C_{r}$ and $C_{c}$ have sons $C_{r 1}, C_{r 2}$ and $C_{c l}, C_{c 2} \underset{\sim}{\text { in T, }}$, respectively, then for all $\left(\tilde{C}_{r}, \tilde{C}_{c}\right)$ in combinations $\left(C_{r 1}, C_{r 2}\right) \times\left(C_{c 1}, C_{c 2}\right)$, set $C_{r} \stackrel{=}{=} \tilde{C}_{r}$ and $C_{c}=\tilde{C}_{c}$ and go to 2 ; otherwise set the submatrices whose row indices and column indices correspond to $C_{r}$ and $C_{c}$ to be inadmissible blocks, respectively.

By the above two procedures, we obtain a coefficient matrix which is hierarchically divided into some submatrices and each submatrix is classified into an admissible or inadmissible block.

3.2.2 Low rank approximation with the ACA

An arbitrary matrix $A \in C^{m \times n}$ can be approximated by two matrices $U \in C^{m \times k}$ and $V \in C^{n \times k}(k<\operatorname{rank} A)$ as

$$
A \simeq U V^{H}
$$

and this is called a low rank approximation of a matrix. In this study, the adaptive cross approximation (ACA) is employed for the low rank approximation. In the ACA, we can obtain $U, V$ and their rank $k$ such that they satisfy the following condition with a given tolerance $\varepsilon_{\mathrm{ACA}}$ :

$$
\left\|U V^{H}-A\right\|_{F} \leq \varepsilon_{\mathrm{ACA}}\|A\|_{F},
$$

where $\|\cdot\|_{F}$ denotes the Frobenius norm. The details of the algorithm of the ACA and its application to the $\mathcal{H}$-matrix method can be found in [6].

\subsubsection{Efficiency improvement of the $\mathcal{H}$-matrix method}

In the process of the BEM, it is time-consuming not only to solve a system of linear algebraic equations but also to compute entries of its coefficient matrix. Each of the entries is calculated as an integral of the second-order tensors (17) and (18). The most time-consuming part of the calculation is the computation of the Hankel function $H_{n}^{(1)}$ which depends on positions of a collocation point and integration points of a numerical integration but is independent of indices of the tensors. Hence we can reduce the computational time by storing the values of $H_{n}^{(1)}$ and reuse them for all indices when we compute the four entries which indicate influence from the same boundary element to the same collocation point.

In the $\mathcal{H}$-matrix method, however, we do not need all of the four entries to construct a coefficient matrix, because not all the entries are required for low rank approximation in the ACA. In this study, we employ this store-and-reuse strategy only for inadmissible blocks and reduce the computational time to construct the matrix.

\section{NUMERICAL EXAMPLES}

In this section, we demonstrate two numerical examples and confirm the effectiveness of the proposed method. The elastic medium is assumed to be steel (mass density $\rho=7.8 \times 10^{3}[\mathrm{~kg}]$, Young's modulus $E=205[\mathrm{GPa}$ ], Poisson's ratio $v=0.3$ ). The parameters of the $\mathcal{H}$-matrix method are set as $\varepsilon_{\mathrm{ACA}}=10^{-5}, n_{\min }=32, \eta=0.7$, and the LU decomposition is used as a direct solver in the boundary element analysis. All of the linear algebraic operations in the LU decomposition are accelerated by the $\mathcal{H}$-matrix method. The incident wave $u^{\text {in }}$ is set as a plane P-wave propagating in $x_{1}$ direction with the frequency $3[\mathrm{kHz}]$. We set the number of the observation points $M=400$ and distribute them around the fixed design domain $D$ at 


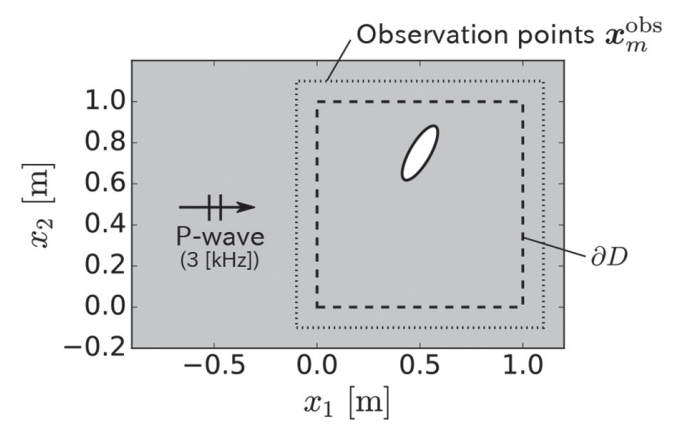

Figure 2: Identification of an elliptical defect.

regular intervals as shown in Fig. 2. The initial level set function $\phi_{0}$ is uniformly set to be 1 in $D$. We first assume that an elliptical defect is allocated in $D$ and use a numerical solution of the BEM, in which each defect is divided into 5,000 boundary elements, as the measured displacement $\hat{u}$. The example numerical calculations are performed on a computer with Intel Xeon CPU E5-1660 with 8 cores, and the code is parallelised using Open MP.

Figures 3 and 4 show results of the optimisations and histories of the objective function $J$ in each case whether the $\mathcal{H}$-matrix method is utilised or not, respectively. ZGETRF routine by LAPACK is used for the LU decomposition in the case that the $\mathcal{H}$-matrix method is not used. Note that $J$ is normalised by the amplitude of the plane incident wave. We see that the results are almost the same and give us a good estimation of the position and shape of the defect. Also, Fig. 5 shows computational time for one optimisation step versus the number of boundary elements in the step. From this comparison, we confirm that the $\mathcal{H}$-matrix method can reduce the computational time for the sensitivity analysis without losing its accuracy.

Next, we add a cylindrical defect to the previous configuration and perform the optimisation as shown in Fig. 6. The result of the optimisation is shown in Fig. 7 and indicates that we

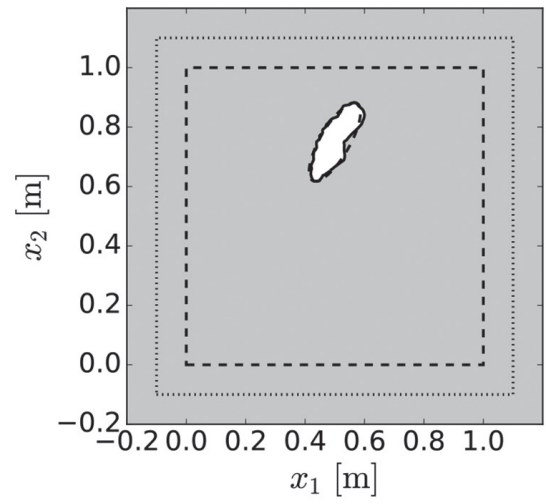

(a) BEM without $\mathcal{H}$-matrix method

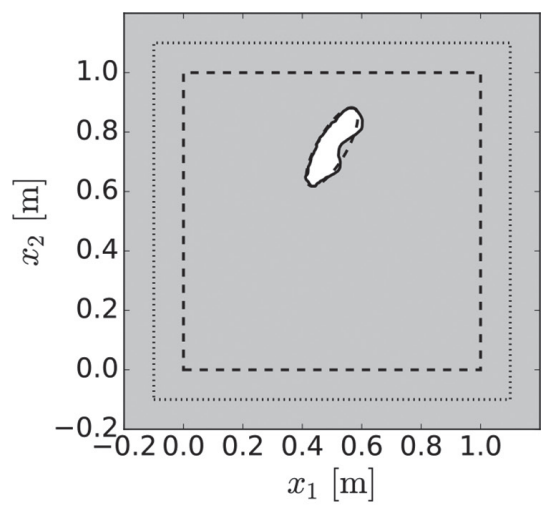

(b) BEM with $\mathcal{H}$-matrix method

Figure 3: Optimal configurations in the case of one elliptical defect. 


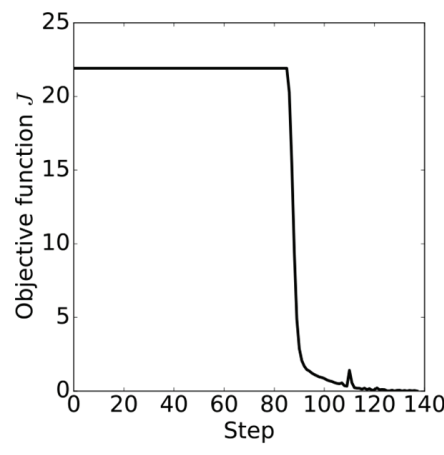

(a) BEM without $\mathcal{H}$-matrix method

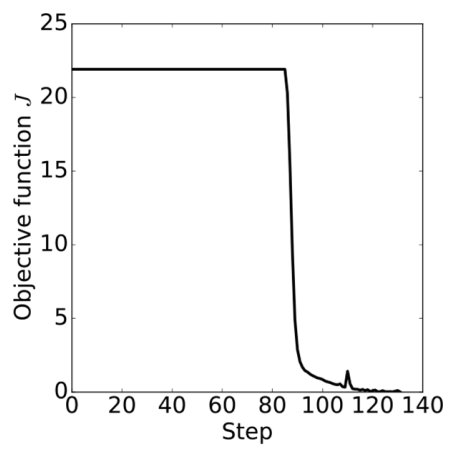

(b) BEM with $\mathcal{H}$-matrix method

Figure 4: Histories of the objective function $J$ in the case of one elliptical defect.

- $\quad$ BEM without $\mathcal{H}$-matrix method - $\quad$ BEM with $\mathcal{H}$-matrix method (proposed)

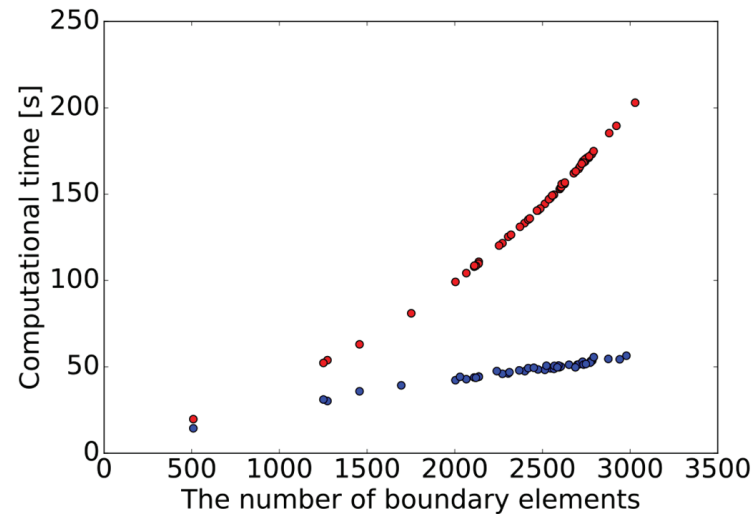

Figure 5: Computational time for one optimisation step.

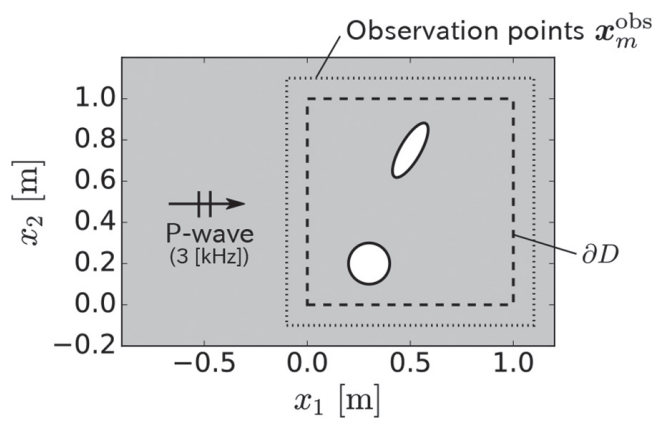

Figure 6: Identification of elliptical and cylindrical defects. 


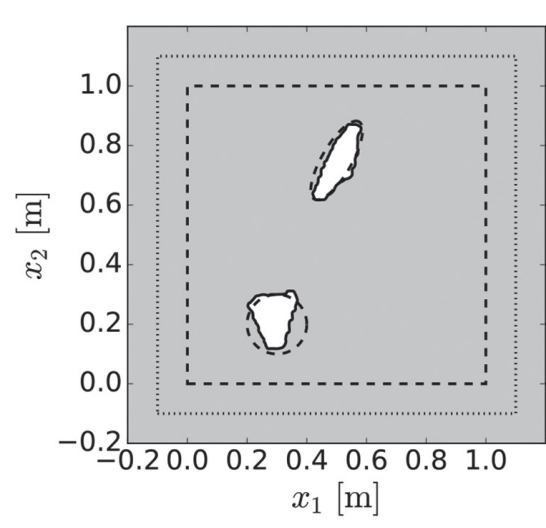

(a) Optimal configuration

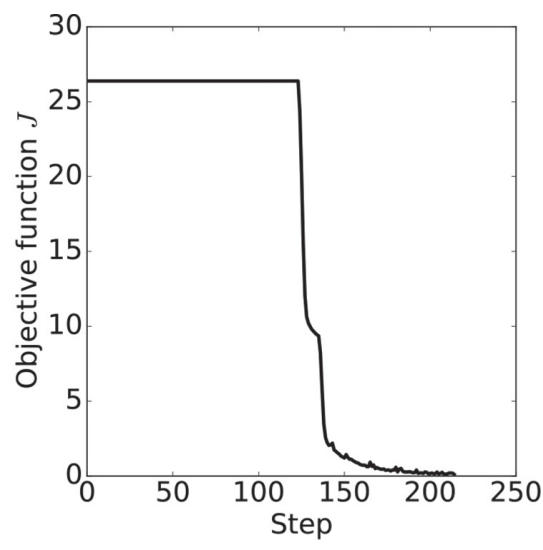

(b) History of the objective function $J$

Figure 7: Optimisation result in the case of elliptical and cylindrical defects.

can identify not only one but two defects though it seems to be less accurate than the case of one defect.

\section{CONCLUSION}

We have developed a numerical method for defect identification in an elastic medium based on the level set method and the BEM. We have employed the $\mathcal{H}$-matrix method to the BEM and reduce the computational cost. Finally, we have demonstrated numerical examples and confirmed that the proposed method is effective for the inverse scattering problem.

\section{ACKNOWLEDGEMENT}

This work was supported by JSPS KAKENHI Grant Number JP16H04255 and Number JP17K14146.

\section{REFERENCES}

[1] Bonnet, M. \& Constantinescu, A., Inverse problems in elasticity. Inverse Problems, 21(2), pp. R1-R50, 2005. https://doi.org/10.1088/0266-5611/21/2/r01

[2] Guzina, B.B. \& Bonnet, M., Topological derivative for the inverse scattering of elastic waves. The Quarterly Journal of Mechanics and Applied Mathematics, 57(2), pp. 161-179, 2004.

https://doi.org/10.1093/qjmam/57.2.161

[3] Bonnet, M. \& Guzina, B.B., Sounding of finite solid bodies by way of topological derivative. International Journal for Numerical Methods in Engineering, 61(13), pp. 2344-2373, 2004. https://doi.org/10.1002/nme.1153

[4] Greengard, L. \& Rokhlin, V., A fast algorithm for particle simulations. Journal of Computational Physics, 73(2), pp. 325-348, 1987.

https://doi.org/10.1016/0021-9991(87)90140-9 
[5] Martinsson, P.G. \& Rokhlin, V., A fast direct solver for boundary integral equations in two dimensions. Journal of Computational Physics, 205(1), pp. 1-23, 2005. https://doi.org/10.1016/j.jcp.2004.10.033

[6] Bebendorf, M., Hierarchical Matrices. Springer Berlin: Heidelberg, 2008.

[7] Nakamoto, K., Isakari, H., Takahashi, T. \& Matsumoto, T., Acceleration of a multi-objective topology optimisation in 2D electro-magnetic field based on the level-set method and the boundary element method by the- $\mathcal{H}$ Matrix Method. International Journal of Computational Methods and Experimental Measurements, 5(5), pp. 686-695, 2017.

[8] Yamada, T., Izui, K., Nishiwaki, S. \& Takezawa, A., A topology optimization method based on the level set method incorporating a fictitious interface energy. Computer Methods in Applied Mechanics and Engineering, 199(45), pp. 2876-2891, 2010. https://doi.org/10.1016/j.cma.2010.05.013

[9] Isakari, H., Kuriyama, K., Harada, S., Yamada, T., Takahashi, T. \& Matsumoto, T., A topology optimisation for three-dimensional acoustics with the level set method and the fast multipole boundary element method. Mechanical Engineering Journal, 1(4), pp. CM0039-CM0039, 2014. https://doi.org/10.1016/j.jcp.2004.10.033

[10] Isakari, H., Nakamoto, K., Kitabayashi, T., Takahashi, T. \& Matsumoto, T., A multiobjective topology optimisation for $2 \mathrm{D}$ electro-magnetic wave problems with the level set method and BEM. European Journal of Computational Mechanics, 25(1-2), pp. 165-193, 2016.

[11] Burton, A.J. \& Miller, G.F., The application of integral equation methods to the numerical solution of some exterior boundary-value problems. Proceedings of the Royal Society of London A: Mathematical, Physical and Engineering Sciences, The Royal Society, 323, pp. 201-210, 1971.

https://doi.org/10.1098/rspa.1971.0097 